\title{
NO Migratory Insertion into Ruthenium-Aryl Bond with a Hydridotris(pyrazolyl)borato Ligand
}

\author{
Yasuhiro Arikawa, ${ }^{*}$ Motoki Yamada, Nobuko Takemoto, Shinnosuke Horiuchi, Eri Sakuda, and \\ Keisuke Umakoshi
}

Division of Chemistry and Materials Science, Graduate School of Engineering, Nagasaki University, Bunkyo-machi 1-14, Nagasaki 852-8521, Japan.

\section{Contents}

Experimental Procedures

Figure S1. Kinetic experiments of insertion reaction of $\mathbf{2}$ with dmap under pseudo-first-order conditions. (Left) Plot of $\ln [2]$ vs time. (Right) Plot of $k_{\text {obs }}$ vs [dmap].

Figure S2. Kinetic experiments of insertion reaction of $\mathbf{2}$ with dmap under pseudo-first-order conditions. (Left) Plot of $\ln [2]$ vs time. (Right) Plot of $\ln \left(k_{\mathrm{obs}} / \mathrm{T}\right)$ vs $1 / \mathrm{T}$ with linear least-squares fit.

Figure S3. ${ }^{1} \mathrm{H}$ NMR spectrum (300 $\left.\mathrm{MHz}, \mathrm{CDCl}_{3}\right)$ of $[\mathrm{TpRuCl}(\mathrm{Ph})(\mathrm{NO})](\mathbf{2})$.

Figure S4. ${ }^{13} \mathrm{C}\left\{{ }^{1} \mathrm{H}\right\}$ NMR spectrum $\left(400 \mathrm{MHz}, \mathrm{CDCl}_{3}\right)$ of $[\mathrm{TpRuCl}(\mathrm{Ph})(\mathrm{NO})](\mathbf{2}) . \quad$ S5

Figure S5. ${ }^{1} \mathrm{H}$ NMR spectrum $\left(300 \mathrm{MHz}, \mathrm{CDCl}_{3}\right)$ of $\left[\mathrm{TpRu}(\mathrm{Ph})_{2}(\mathrm{NO})\right](\mathbf{3}) . \quad$ S6

Figure S6. ${ }^{13} \mathrm{C}\left\{{ }^{1} \mathrm{H}\right\}$ NMR spectrum $\left(400 \mathrm{MHz}, \mathrm{CDCl}_{3}\right)$ of $\left[\mathrm{TpRu}(\mathrm{Ph})_{2}(\mathrm{NO})\right](\mathbf{3}) . \quad$ S6

Figure S7. ${ }^{1} \mathrm{H}$ NMR spectrum $\left(300 \mathrm{MHz}, \mathrm{CDCl}_{3}\right)$ of $[\mathrm{TpRuCl}(\mathrm{dmap})\{\mathrm{N}(=\mathrm{O}) \mathrm{Ph}\}](4) . \quad \mathrm{S} 7$

Figure S8. ${ }^{13} \mathrm{C}\left\{{ }^{1} \mathrm{H}\right\}$ NMR spectrum $\left(400 \mathrm{MHz}, \mathrm{CDCl}_{3}\right)$ of $[\mathrm{TpRuCl}(\mathrm{dmap})\{\mathrm{N}(=\mathrm{O}) \mathrm{Ph}\}](\mathbf{4}) . \quad \mathrm{S} 7$

$\mathrm{X}$-ray Crystal Structure Determinations

Table S1. Crystallographic data for $[\mathrm{TpRuCl}(\mathrm{Ph})(\mathrm{NO})](\mathbf{2}),\left[\mathrm{TpRu}(\mathrm{Ph})_{2}(\mathrm{NO})\right](\mathbf{3})$, and $[\mathrm{TpRuCl}(\mathrm{dmap})\{\mathrm{N}(=\mathrm{O}) \mathrm{Ph}\}]$ (4). 


\section{Experimental Procedures}

General Procedures: All reactions were carried out under $\mathrm{N}_{2}$ or Ar unless otherwise noted and subsequent work-up manipulations were performed in air. The starting material $\left[\mathrm{TpRuCl}_{2}(\mathrm{NO})\right]$ $(\mathbf{1})^{1}$ was prepared according to the previously reported method. Reaction solvent (THF) was passed over columns of activated alumina and a supported copper catalyst supplied by Hansen \& Co. Ltd. Other organic solvents and all other reagents were commercially available and used without further purification, including $\mathrm{ZnPh}_{2}$ (Sigma-Aldrich Co. LLC or STREM Chemicals, Inc.). NMR spectra were recorded on a Varian Gemini-300, a Varian NMR System 500PS SN, and a JEOL JNM-AL-400 spectrometers. ${ }^{1} \mathrm{H}$ and ${ }^{13} \mathrm{C}\left\{{ }^{1} \mathrm{H}\right\}$ NMR chemical shifts are quoted with respect to TMS and the solvent signals, respectively. Infrared spectra in $\mathrm{KBr}$ pellets were obtained on JASCO FT-IR-4100 spectrometers. Electron ionization mass spectra (EI MS) and fast atom bombardment mass spectra (FAB MS) were recorded on a JEOL JMS-700N spectrometer. Elemental analyses $(\mathrm{C}, \mathrm{H}, \mathrm{N})$ were performed on a Perkin Elmer 2400II elemental analyzer.

Preparations of [TpRuCl(Ph)(NO)] (2) and [TpRu(Ph) $2(\mathrm{NO})]$ (3): Diphenylzinc (0.131 g, $0.60 \mathrm{mmol})$ in dried THF $(2 \mathrm{~mL})$ was added dropwise to a dried THF $(8.0 \mathrm{~mL})$ solution of $\left[\mathrm{TpRuCl}_{2}(\mathrm{NO})\right](\mathbf{1})(50.4 \mathrm{mg}, 0.12 \mathrm{mmol})$ at $-80{ }^{\circ} \mathrm{C}$ for $1 \mathrm{~h}$. The reaction mixture was stirred under gradual warming (to ca. $4^{\circ} \mathrm{C}$ ) for another $3 \mathrm{~h}$. After addition of methanol to quench any reactive diphenylzinc species, the mixture was evaporated to dryness. The residue was extracted with $\mathrm{CH}_{2} \mathrm{Cl}_{2}$ and filtered to produce a red-purple solution. Column chromatographic separation of the $\mathrm{CH}_{2} \mathrm{Cl}_{2}$ extract was performed on a silica gel with $\mathrm{CH}_{2} \mathrm{Cl}_{2}$ elution to give a monophenyl complex $[\mathrm{TpRuCl}(\mathrm{Ph})(\mathrm{NO})]$ (2) in $52 \%$ yield $(28.4 \mathrm{mg}$ ) along with a diphenyl complex $\left[\mathrm{TpRu}(\mathrm{Ph})_{2}(\mathrm{NO})\right](3)$ and biphenyl. The yield of $\mathbf{3}$ was $5.7 \%(3.4 \mathrm{mg})$ based on the ${ }^{1} \mathrm{H}$ NMR spectrum due to low yield and inseparable with biphenyl.

To a dried THF $(8.0 \mathrm{~mL})$ solution of the isolated complex $2(31.4 \mathrm{mg}, 0.069 \mathrm{mmol})$ was added diphenylzinc $(0.131 \mathrm{~g}, 0.60 \mathrm{mmol})$ in dried THF $(2 \mathrm{~mL})$. The reaction mixture was stirred under reflux for $11 \mathrm{~h}$. After addition of methanol to quench any reactive diphenylzinc species, the mixture was evaporated to dryness. The residue was extracted with $\mathrm{CH}_{2} \mathrm{Cl}_{2}$ and filtered. Column chromatographic separation of the $\mathrm{CH}_{2} \mathrm{Cl}_{2}$ extract was performed on a silica gel with $\mathrm{CH}_{2} \mathrm{Cl}_{2}$ elution to give a red-purple powder, followed by recrystallization from benzene/n-pentane to afford the diphenyl complex 3 in $39 \%$ yield (13.4 mg).

Complex 2: $\quad$ IR (KBr): v(BH) $2497(\mathrm{~m}), v(\mathrm{NO}) 1854(\mathrm{~s}) \mathrm{cm}^{-1} . \quad{ }^{1} \mathrm{H}$ NMR: $\delta 8.08(\mathrm{~d}, 1 \mathrm{H}, J=1.9$ $\mathrm{Hz}, \mathrm{pz}), 7.88(\mathrm{~d}, 1 \mathrm{H}, J=2.4 \mathrm{~Hz}, \mathrm{pz}), 7.77(\mathrm{~d}, 1 \mathrm{H}, J=2.3 \mathrm{~Hz}, \mathrm{pz}), 7.61(\mathrm{~d}, 1 \mathrm{H}, J=2.9 \mathrm{~Hz}, \mathrm{pz}), 7.40$ $(\mathrm{d}, 1 \mathrm{H}, J=2.2 \mathrm{~Hz}, \mathrm{pz}), 7.30(\mathrm{~d}, 1 \mathrm{H}, J=2.1 \mathrm{~Hz}, \mathrm{pz}), 7.16-7.04(\mathrm{~m}, 5 \mathrm{H}$, phenyl), $6.40(\mathrm{t}, 1 \mathrm{H}, J=2.2$ $\mathrm{Hz}, 4-\mathrm{pz}), 6.38$ (t, 1H, $J=2.4 \mathrm{~Hz}, 4-\mathrm{pz}), 6.12(\mathrm{t}, 1 \mathrm{H}, J=2.3 \mathrm{~Hz}, 4-\mathrm{pz}) . \quad{ }^{13} \mathrm{C}\left\{{ }^{1} \mathrm{H}\right\} \mathrm{NMR}: \delta 155.5$ (ipso-Ph), 144.8 (pz), 143.5 (pz), 142.4 (pz), 138.3 (Ph), 136.6 (pz), 135.4 (pz), 134.8 (pz), 127.3 
(Ph), 124.7 (Ph), 107.4 (4-pz), 106.6 (4-pz), 105.9 (4-pz). EI MS (m/z): $457\left([M]^{+}\right), 427$ $\left([M-(\mathrm{NO})]^{+}\right), 380\left([M-\mathrm{Ph}]^{+}\right), 345\left([M-\mathrm{Cl}-\mathrm{Ph}]^{+}\right), 315\left([\mathrm{TpRu}]^{+}\right)$. Elemental analysis $(\%)$ calcd for $\mathrm{C}_{15} \mathrm{H}_{15} \mathrm{~N}_{7}$ BClORu: C 39.45, H 3.31, N 21.47; found: C 39.44, H 2.86, N 21.03.

Complex 3: IR (KBr): v(BH) $2485(\mathrm{~m}), v(\mathrm{NO}) 1845$ (s), $1831(\mathrm{~s}) \mathrm{cm}^{-1} . \quad{ }^{1} \mathrm{H}$ NMR: $\delta 7.77$ (d, 2H, $J=2.3 \mathrm{~Hz}, \mathrm{pz}), 7.63(\mathrm{~d}, 1 \mathrm{H}, J=2.3 \mathrm{~Hz}, \mathrm{pz}), 7.39$ (d, 2H, $J=1.8 \mathrm{~Hz}, \mathrm{pz}), 7.17-7.14(\mathrm{~m}, 4 \mathrm{H}$, phenyl), 7.10 (d, 1H, $J=2.0 \mathrm{~Hz}, \mathrm{pz}), 7.07-6.97$ (m, 6H, phenyl), 6.26 (t, 2H, $J=2.0 \mathrm{~Hz}, 4-\mathrm{pz}$ ), $6.09(\mathrm{t}, 1 \mathrm{H}, J=2.2 \mathrm{~Hz}, 4-\mathrm{pz}) . \quad{ }^{13} \mathrm{C}\left\{{ }^{1} \mathrm{H}\right\} \mathrm{NMR}: \delta 159.8$ (ipso-Ph), $145.6(\mathrm{pz}), 142.5(\mathrm{pz}), 138.0$ $(\mathrm{Ph}), 135.2(\mathrm{pz}), 135.1(\mathrm{pz}), 127.0(\mathrm{Ph}), 123.6(\mathrm{Ph}), 106.1$ (4-pz), $105.5(4-\mathrm{pz}) . \quad$ EI MS (m/z): 499 $\left([M]^{+}\right), 469\left([M-(\mathrm{NO})]^{+}\right), 345\left(\left[M-(\mathrm{Ph})_{2}\right]^{+}\right), 315\left([\mathrm{TpRu}]^{+}\right)$. Elemental analysis $(\%)$ calcd for $\mathrm{C}_{21} \mathrm{H}_{20} \mathrm{~N}_{7} \mathrm{BORu}$ : C 50.62, H 4.05, N 19.68; found: C 50.78, H 3.84, N 19.34.

Reaction of [TpRuCl(Ph)(NO)] (2) with DMAP (4-dimethylaminopyridine): A mixture of complex 2 (22.8 $\mathrm{mg}, 0.050 \mathrm{mmol})$ and DMAP $(60.9 \mathrm{mg}, 0.50 \mathrm{mmol})$ in toluene $(5 \mathrm{~mL})$ was refluxed for 5.5 days. After evaporation, the residue was purified by column chromatography with a silica gel using a $\mathrm{CH}_{2} \mathrm{Cl}_{2}$ /acetone (20/1) eluent to give a nitrosobenzene complex $[\mathrm{TpRuCl}(\mathrm{dmap})\{\mathrm{N}(=\mathrm{O}) \mathrm{Ph}\}]$ (4) in $67 \%$ yield $(19.4 \mathrm{mg})$.

Complex 4: $\quad \mathrm{IR}(\mathrm{KBr}): v(\mathrm{BH}) 2481(\mathrm{w}) \mathrm{cm}^{-1} . \quad{ }^{1} \mathrm{H}$ NMR: $\delta 8.08(\mathrm{~d}, J=7.4 \mathrm{~Hz}, 2 \mathrm{H}, \mathrm{dmap}), 7.96(\mathrm{~d}$, $J=1.7 \mathrm{~Hz}, 1 \mathrm{H}, \mathrm{pz}), 7.83(\mathrm{~d}, J=1.6 \mathrm{~Hz}, 1 \mathrm{H}, \mathrm{pz}), 7.78(\mathrm{~d}, J=1.7 \mathrm{~Hz}, 1 \mathrm{H}, \mathrm{pz}), 7.44$ (d, $J=1.5 \mathrm{~Hz}$, 1H, pz), 7.33-7.27 (m, 1H, Ph), 6.94-6.85 (m, 4H, Ph), 6.88 (d, $J=2.3 \mathrm{~Hz}, 1 \mathrm{H}, \mathrm{pz}), 6.38$ (d, $J=$ $7.4 \mathrm{~Hz}, 2 \mathrm{H}$, dmap), 6.30 (d, $J=1.5 \mathrm{~Hz}, 1 \mathrm{H}, \mathrm{pz}), 6.23$ (t, $J=2.2 \mathrm{~Hz}, 1 \mathrm{H}, 4-\mathrm{pz}), 6.11$ (t, $J=2.3 \mathrm{~Hz}$, 1H, 4-pz), 6.00 (t, $J=2.3 \mathrm{~Hz}, 1 \mathrm{H}, 4-\mathrm{pz}), 3.01$ (s, 6H, NMe $) .{ }^{13} \mathrm{C}\left\{{ }^{1} \mathrm{H}\right\} \mathrm{NMR}: \delta 167.5$ (ipso-Ph), 154.2 (ipso-dmap), 153.4 (dmap), $144.4(\mathrm{Ph}), 144.1$ (pz), 143.0 (pz), 136.5 (pz), 135.7 (pz), 135.3 (pz), 129.3 (pz), 127.8 (Ph), 120.9 (Ph), 106.8 (4-pz), 106.5 (4-pz), 106.3 (4-pz), 106.2 (dmap), $39.0\left(\mathrm{~N} M e_{2}\right) . \quad$ FAB $\quad$ MS $(m / z): 579\left([M]^{+}\right), 544\left([M-\mathrm{Cl}]^{+}\right), 472\left([M-\{\mathrm{N}(=\mathrm{O}) \mathrm{Ph}\}]^{+}\right), 422$ $\left([\mathrm{TpRu}\{\mathrm{N}(=\mathrm{O}) \mathrm{Ph}\}]^{+}\right), \quad 350 \quad\left([\mathrm{TpRu}]^{+}\right) . \quad$ Elemental analysis $(\%)$ calcd for $\mathrm{C}_{22} \mathrm{H}_{25} \mathrm{~N}_{9} \mathrm{BClORu} \cdot\left(\mathrm{C}_{6} \mathrm{H}_{6}\right)_{0.5}$ : C 48.60, H 4.57, N 20.40; found: C 48.92, H 4.15, N 20.26.

Kinetic experiments: Kinetic experiments were carried out under pseudo-first-order conditions, using a large excess amount of DMAP. Complex 2 (2.74 mg, $10 \mathrm{mM})$, DMAP (10 eq., 25 eq., 50 eq., 75 eq. or 100 eq.), and hexamethylbenzene (1.00 mg; the internal standard) were dissolved in toluene- $\mathrm{d}_{8}(0.60 \mathrm{~mL})$. The solution was transferred to a J-Young NMR tube and the conversion of 2 to 3 were monitored with ${ }^{1} \mathrm{H}$ NMR at $100^{\circ} \mathrm{C}$. The concentration of 2 were obtained from the NMR signal integral ratios between 2 and the internal standard.

Plotting $\ln [2]$ against time show linear relationships, indicating that the reactions are first order in [2] (Figure S1). Pseudo-first-order rate constants $\left(k_{\mathrm{obs}}\right)$ were determined from the slope of these lines by the least-squares method. A plot of the $k_{\text {obs }}$ values vs. [dmap] shows that a 10-fold 
increase in [dmap] produces only a very weak response in $k_{\text {obs }}$ (Figure $\mathrm{S} 1$ ), indicating the rate-determining formation of an intermediate. Using 10 eq. of DMAP, five rate constants were determined in the temperature range of $80^{\circ} \mathrm{C}$ to $100^{\circ} \mathrm{C}$ at $5^{\circ} \mathrm{C}$ intervals (Figure S2). A plot of $\ln \left(k_{\text {obs }} / \mathrm{T}\right)$ against $1 / \mathrm{T}$ allows calculation of the thermodynamic parameters using the Eyring equation (Figure S2). The activation parameters were found to be: $\Delta H^{\ddagger}=+18 \pm 2 \mathrm{kcal} \mathrm{mol}^{-1}, \Delta S^{\ddagger}$ $=-36 \pm 4 \mathrm{cal} \mathrm{K}^{-1} \mathrm{~mol}^{-1}$ and an associated $\Delta G^{\ddagger}(298 \mathrm{~K})=+29 \pm 2 \mathrm{kcal} \mathrm{mol}^{-1}$.
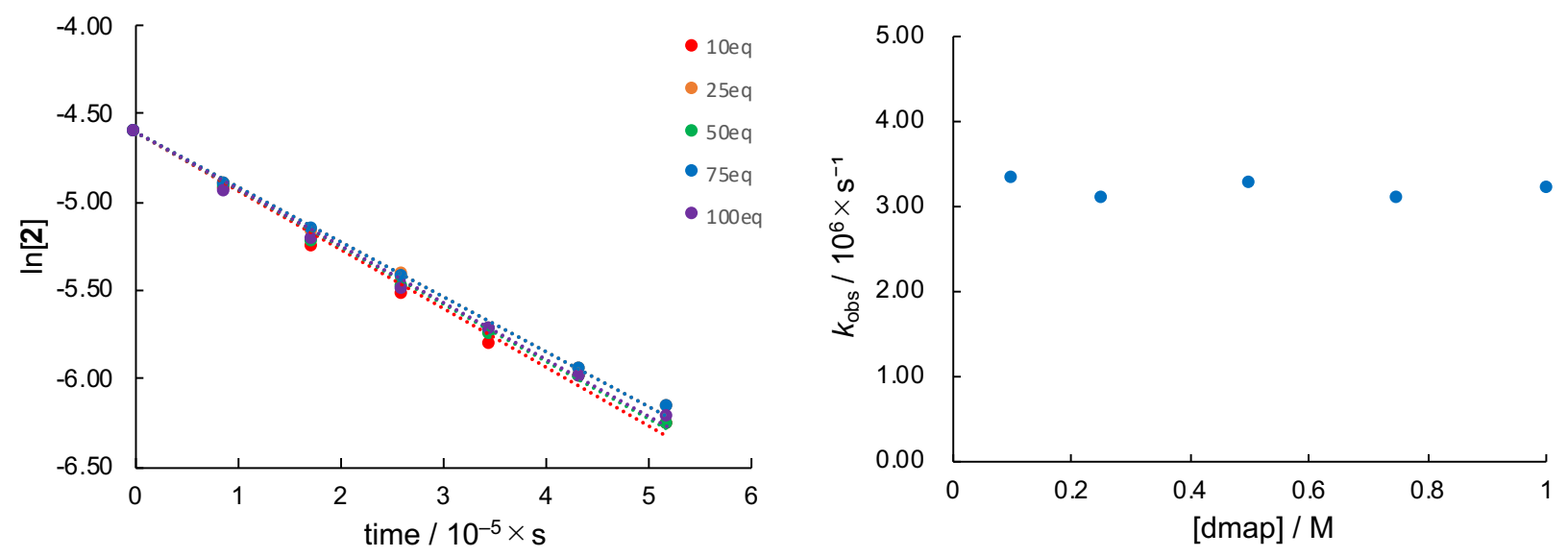

Figure S1. Kinetic experiments of insertion reaction of 2 with dmap under pseudo-first-order conditions. (Left) Plot of $\ln [2]$ vs time. (Right) Plot of $k_{\text {obs }}$ vs [dmap].
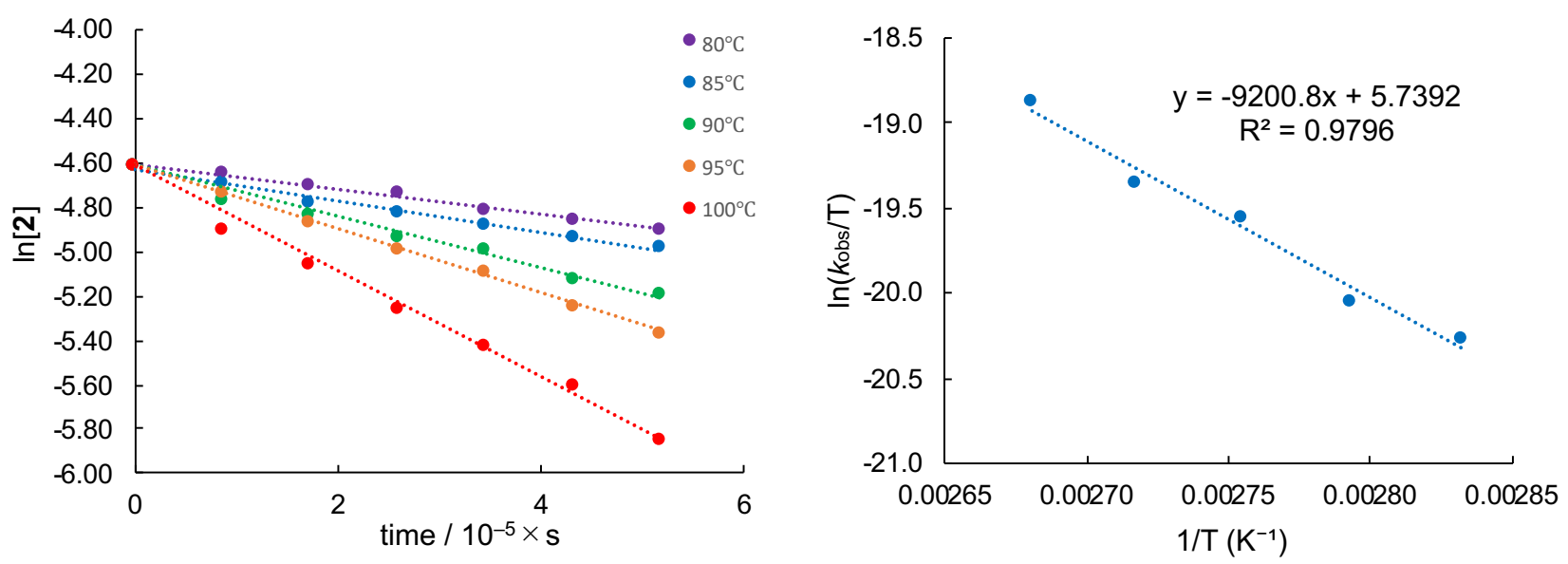

Figure S2. Kinetic experiments of insertion reaction of $\mathbf{2}$ with dmap under pseudo-first-order conditions. (Left) Plot of $\ln [2]$ vs time. (Right) Plot of $\ln \left(k_{\mathrm{obs}} / \mathrm{T}\right)$ vs $1 / \mathrm{T}$ with linear least-squares fit. 


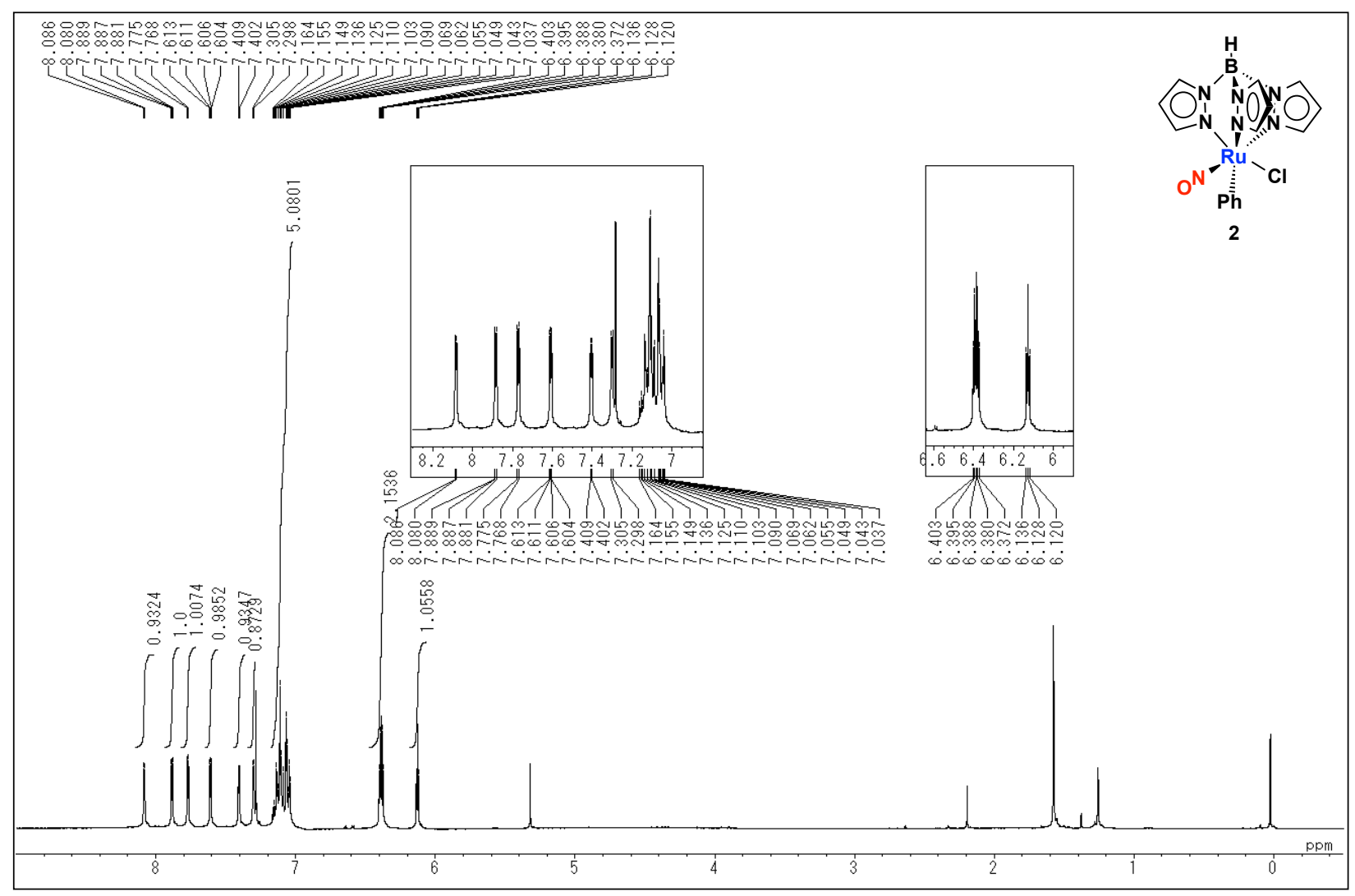

Figure S3. ${ }^{1} \mathrm{H}$ NMR spectrum (300 MHz, $\left.\mathrm{CDCl}_{3}\right)$ of $[\mathrm{TpRuCl}(\mathrm{Ph})(\mathrm{NO})]$ (2).

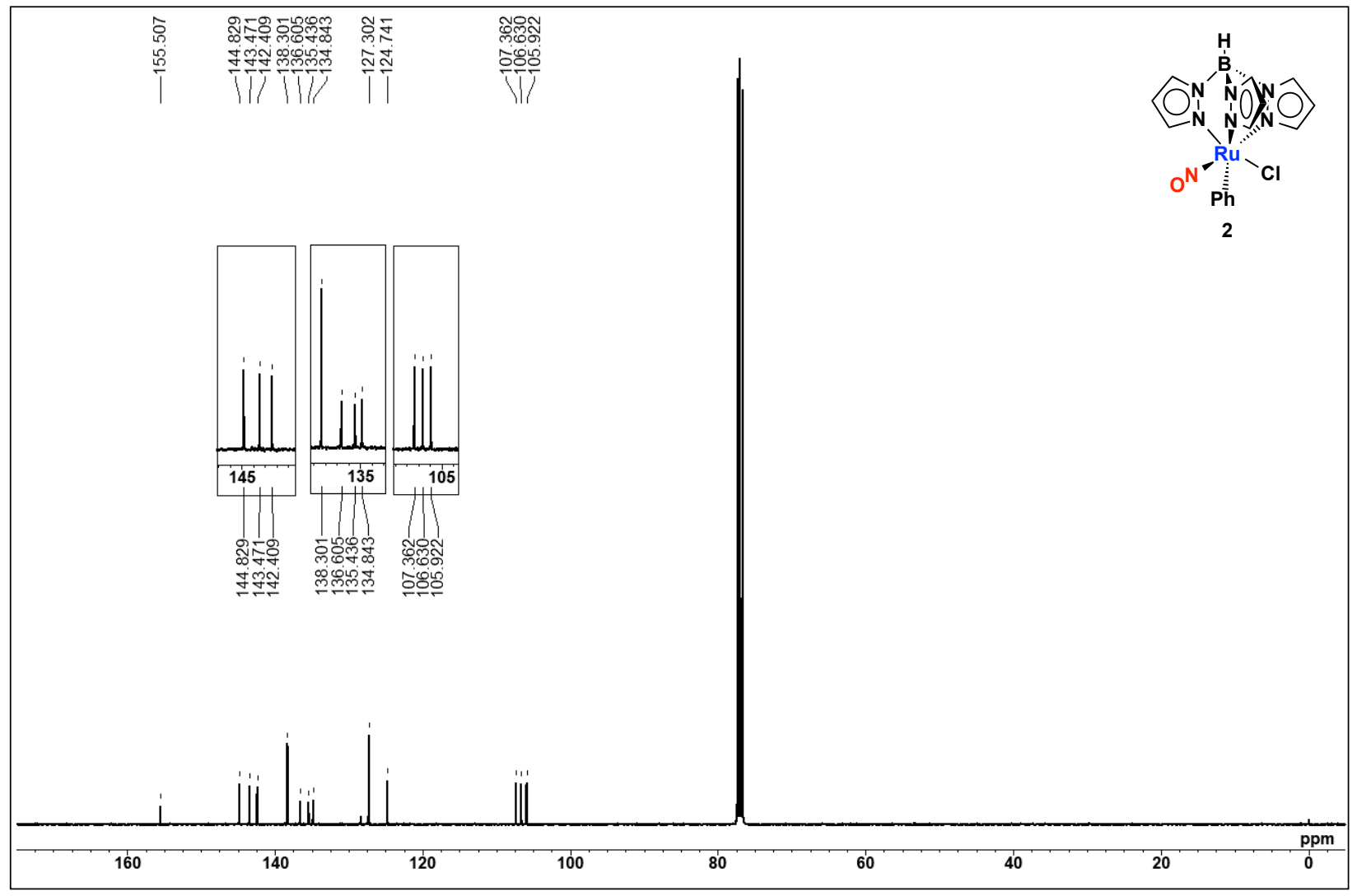

Figure S4. ${ }^{13} \mathrm{C}\left\{{ }^{1} \mathrm{H}\right\}$ NMR spectrum $\left(400 \mathrm{MHz}, \mathrm{CDCl}_{3}\right)$ of $[\mathrm{TpRuCl}(\mathrm{Ph})(\mathrm{NO})]$ (2). 


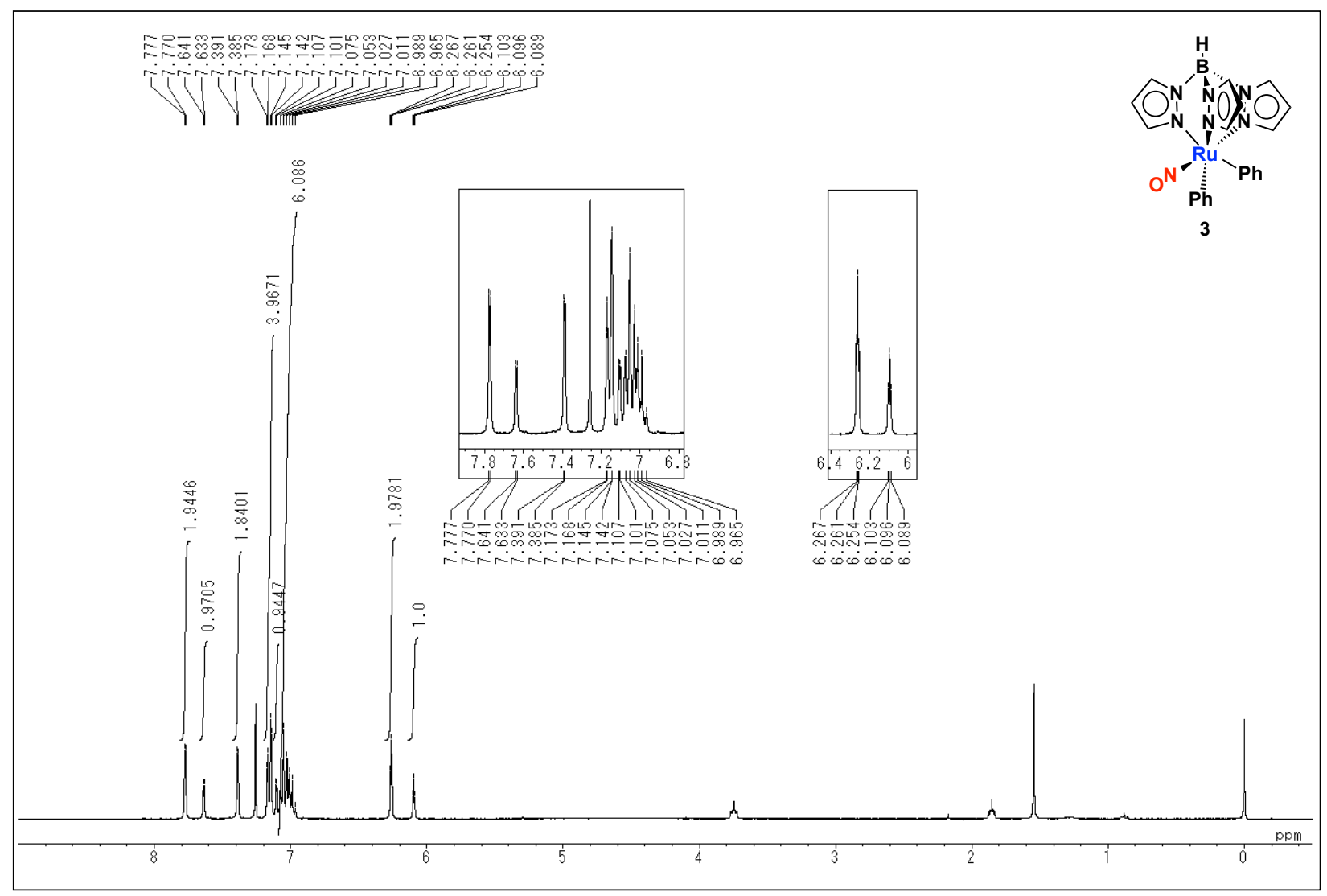

Figure S5. ${ }^{1} \mathrm{H}$ NMR spectrum $\left(300 \mathrm{MHz}, \mathrm{CDCl}_{3}\right)$ of $\left[\mathrm{TpRu}(\mathrm{Ph})_{2}(\mathrm{NO})\right](3)$.

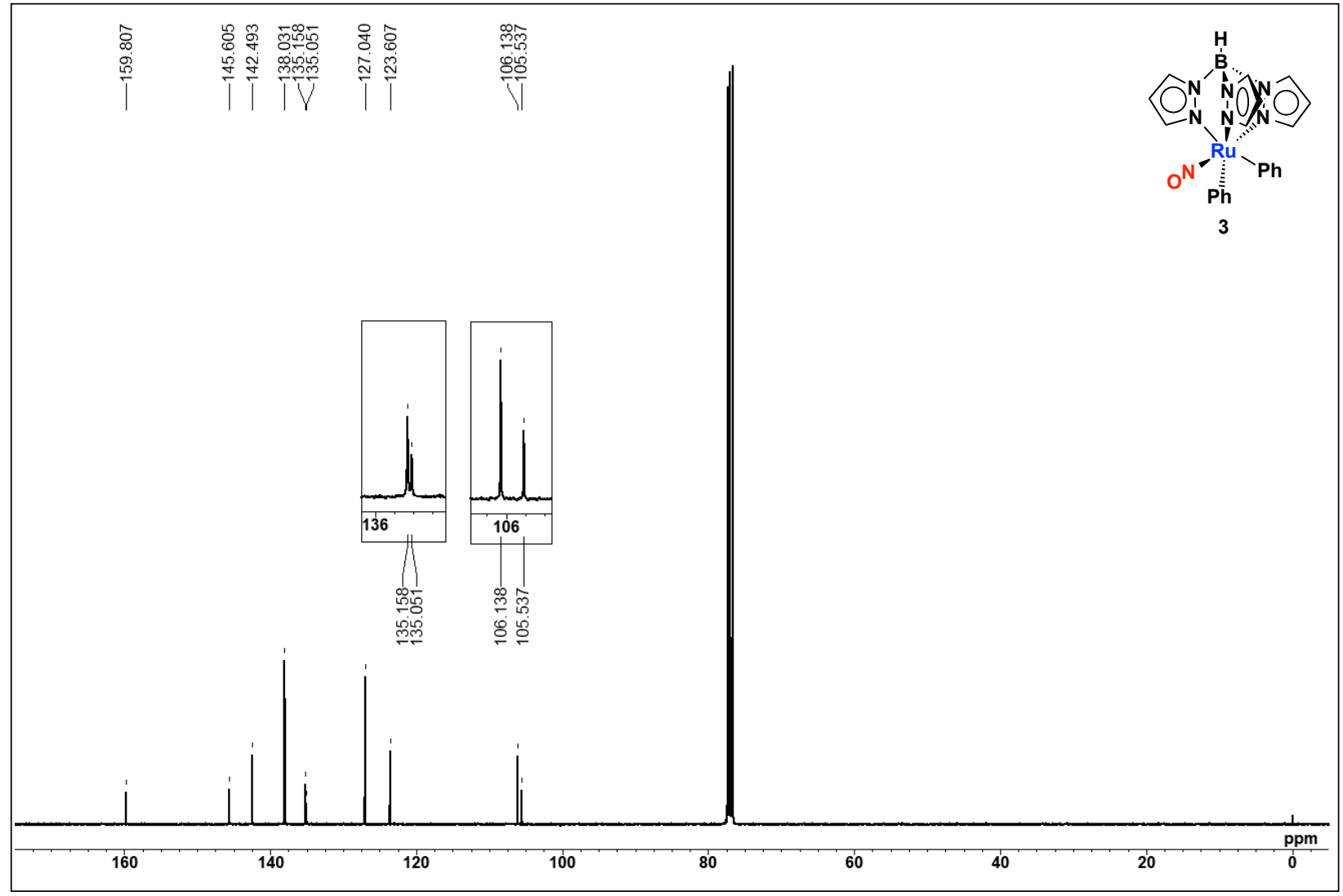

Figure S6. ${ }^{13} \mathrm{C}\left\{{ }^{1} \mathrm{H}\right\}$ NMR spectrum $\left(400 \mathrm{MHz}, \mathrm{CDCl}_{3}\right)$ of $\left[\mathrm{TpRu}(\mathrm{Ph})_{2}(\mathrm{NO})\right](3)$. 


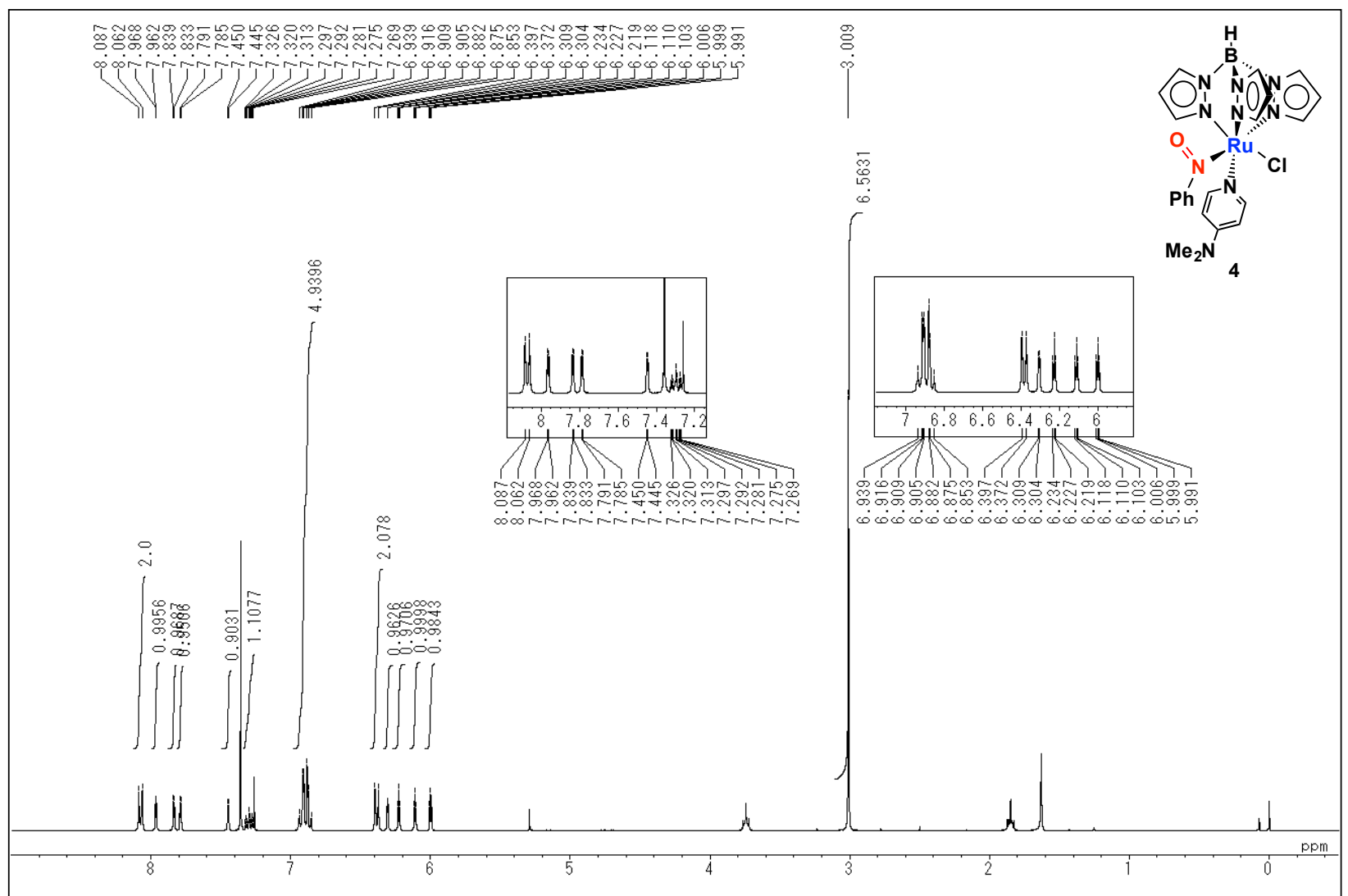

Figure S7. ${ }^{1} \mathrm{H}$ NMR spectrum $\left(300 \mathrm{MHz}, \mathrm{CDCl}_{3}\right)$ of $[\mathrm{TpRuCl}(\mathrm{dmap})\{\mathrm{N}(=\mathrm{O}) \mathrm{Ph}\}](4)$.

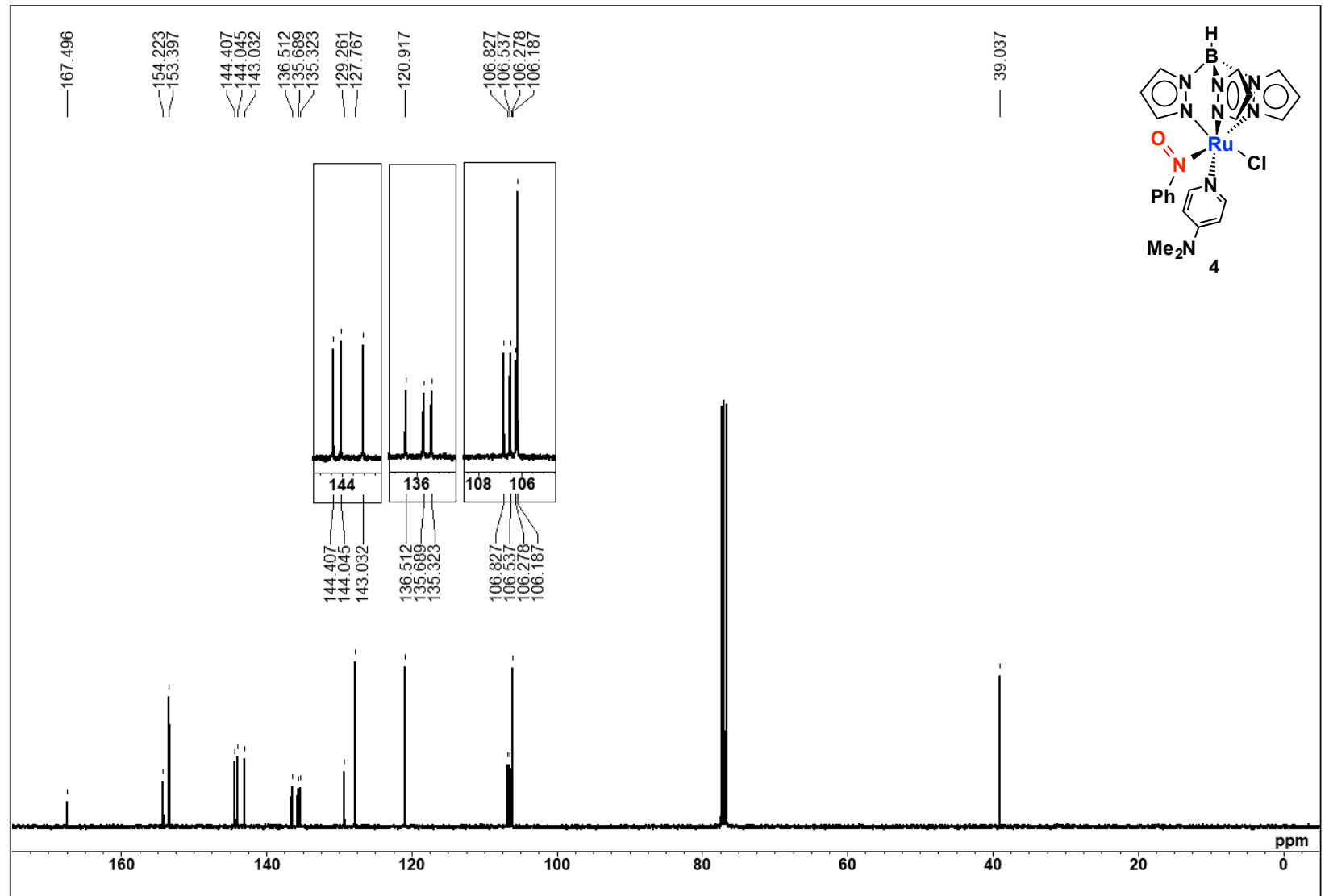

Figure S8. ${ }^{13} \mathrm{C}\left\{{ }^{1} \mathrm{H}\right\}$ NMR spectrum $\left(400 \mathrm{MHz}, \mathrm{CDCl}_{3}\right)$ of $[\mathrm{TpRuCl}(\mathrm{dmap})\{\mathrm{N}(=\mathrm{O}) \mathrm{Ph}\}](4)$. 


\section{X-ray Crystal Structure Determinations}

Crystallographic data are summarized in Table S1. X-ray quality single crystals were obtained from $\mathrm{CH}_{2} \mathrm{Cl}_{2} /$ pentane (for 2), THF/pentane (for $\mathbf{3} \cdot(\text { thf })_{0.25}$ ), and benzene/pentane (for $\left.\mathbf{4} \cdot\left(\mathrm{C}_{6} \mathrm{H}_{6}\right)_{0.5}\right)$, respectively. Diffraction data were collected at $-180{ }^{\circ} \mathrm{C}$ under a stream of cold dinitrogen gas on a Rigaku AFC10 diffractometer equipped with a Rigaku Saturn CCD area detector by using graphite-monochromated Mo K $\alpha$ radiation. The intensity images were obtained at the exposure of $16 \mathrm{~s} /{ }^{\circ}\left(\mathbf{2}\right.$ and $\left.\mathbf{4} \cdot\left(\mathrm{C}_{6} \mathrm{H}_{6}\right)_{0.5}\right)$ or $8 \mathrm{~s} /{ }^{\circ}\left(\mathbf{3} \cdot(\text { thf })_{0.25}\right)$. The frame data were integrated using a Rigaku CrystalClear program package, and the data sets were corrected for absorption using a REQAB program.

The calculations were performed with a CrystalStructure software package. The structures were solved by direct methods except for 3·(thf) $0_{0.25}$ (Patterson Methods) and refined on $F^{2}$ by the full-matrix least squares methods. For $\mathbf{3} \cdot(\text { thf })_{0.25}$, the asymmetric unit contains two crystallographically independent molecules of 3. The thf crystallization solvent was disordered over two positions with occupancy factors of $0.5 / 0.5$. For $4 \cdot\left(\mathrm{C}_{6} \mathrm{H}_{6}\right)_{0.5}$, half of the benzene crystallization solvent was included. Anisotropic refinement was applied to all non-hydrogen atoms, except for the disordered crystallization solvents of $\mathbf{3} \cdot(\text { thf })_{0.25}$. Hydrogen atoms for all structures were put at calculated positions, while those of the disordered crystallization solvents of 3.(thf $)_{0.25}$ were not included in the calculations. 
Table S1. Crystallographic data for $[\mathrm{TpRuCl}(\mathrm{Ph})(\mathrm{NO})] \quad(\mathbf{2}), \quad\left[\mathrm{TpRu}(\mathrm{Ph})_{2}(\mathrm{NO})\right] \quad(\mathbf{3})$, and $[\mathrm{TpRuCl}(\mathrm{dmap})\{\mathrm{N}(=\mathrm{O}) \mathrm{Ph}\}](\mathbf{4})$.

\begin{tabular}{|c|c|c|c|}
\hline & 2 & $\mathbf{3} \cdot(\text { thf })_{0.25}$ & $\mathbf{4} \cdot\left(\mathrm{C}_{6} \mathrm{H}_{6}\right)_{0.5}$ \\
\hline formula & $\mathrm{C}_{15} \mathrm{H}_{15} \mathrm{BClN}_{7} \mathrm{ORu}$ & $\mathrm{C}_{22} \mathrm{H}_{20} \mathrm{BN}_{7} \mathrm{O}_{1.25} \mathrm{Ru}$ & $\mathrm{C}_{25} \mathrm{H}_{28} \mathrm{BClN}{ }_{9} \mathrm{ORu}$ \\
\hline fw & 456.66 & 514.33 & 617.89 \\
\hline cryst system & monoclinic & triclinic & monoclinic \\
\hline space group & $P 2_{1} / c$ (No. 14$)$ & $P-1$ (No. 2) & $P 2_{1} / n$ (No. 14) \\
\hline color of crystal & red purple & purple & orange \\
\hline crystal size (mm) & $0.09 \times 0.08 \times 0.06$ & $0.18 \times 0.18 \times 0.10$ & $0.11 \times 0.06 \times 0.03$ \\
\hline$a(\AA)$ & $11.885(2)$ & $9.6940(17)$ & $10.019(2)$ \\
\hline$b(\AA)$ & $9.8184(16)$ & $10.7348(18)$ & $19.913(4)$ \\
\hline$c(\AA)$ & $15.467(3)$ & $21.909(4)$ & $13.327(3)$ \\
\hline$\alpha(\operatorname{deg})$ & 90 & $91.488(3)$ & 90 \\
\hline$\beta(\operatorname{deg})$ & $95.481(3)$ & $97.040(2)$ & $93.729(3)$ \\
\hline$\gamma(\operatorname{deg})$ & 90 & $102.613(2)$ & 90 \\
\hline$V\left(\AA^{3}\right)$ & $1796.7(5)$ & $2204.8(6)$ & $2653.2(10)$ \\
\hline$Z$ & 4 & 4 & 4 \\
\hline$\rho_{\text {calc }}\left(\mathrm{g} \mathrm{cm}^{-3}\right)$ & 1.688 & 1.549 & 1.547 \\
\hline$\mu\left(\mathrm{cm}^{-1}\right)$ & 10.412 & 7.423 & 7.296 \\
\hline $2 \theta_{\max }(\mathrm{deg})$ & 54.9 & 55.0 & 55.0 \\
\hline no. of all reflns collected & 14542 & 18334 & 21837 \\
\hline no. of unique reflns & 4099 & 9955 & 6062 \\
\hline$R_{\text {int }}$ & 0.0431 & 0.0185 & 0.0531 \\
\hline no. of obsd reflns ${ }^{a}$ & 3470 & 8168 & 4963 \\
\hline no. of parameters & 235 & 575 & 345 \\
\hline$R_{1}^{a, b}$ & 0.0340 & 0.0277 & 0.0500 \\
\hline $\mathrm{w} R_{2}$ (all data) $)^{c}$ & 0.0682 & 0.0787 & 0.1185 \\
\hline GOF (all data) $)^{d}$ & 1.065 & 1.067 & 1.159 \\
\hline CCDC numbers & 2040961 & 2040962 & 2040963 \\
\hline
\end{tabular}




\section{Reference}

1) M. Onishi, Bull. Chem. Soc. Jpn. 1991, 64, 3039 - 3045. 